\title{
REMOTE ATTITUDE SENSING BASED ON HIGH-SPEED MUELLER MATRIX ELLIPSOMETRY
}

\author{
Song Zhang, Hao Jiang *, Honggang Gu *, Xiuguo Chen, Shiyuan Liu
}

State Key Laboratory of Digital Manufacturing Equipment and Technology, Huazhong University of Science and Technology, Wuhan 430074, China - (songzhang, hjiang, hongganggu, xiuguochen, shyliu)@ hust.edu.cn

KEY WORDS: Attitude metrology, Mueller matrix ellipsometry, Birefringence, Real-time remote sensing, Attitude angle

\begin{abstract}
:
The attitude metrology of moving objects has always been a hot topic in engineering applications. In this article, a novel optical method based on the high-speed Mueller matrix ellipsometry (MME) is proposed for real-time remote attitude sensing. With the help of an electric level, all the attitude angles of a free-moving object with a birefringent waveplate attached can be simultaneously extracted in real time. Compared with existing ellipsometry-based attitude metrology that can only be used in the transmission mode, the proposed method with the advantages of the high-speed MME can expand the application to both the transmission and reflection modes while maintaining higher accuracy and robustness in an ultra-large measurement range. The basic principle is presented via theoretical derivation, and the change in the Mueller matrix of the birefringent waveplate versus the attitude angles is explored in the simulated experiments. In a series of the dynamic experiments, with the extremely high temporal resolution and accuracy of the high-speed MME, an angular resolution up to $0.00022^{\circ}$ and an angular accuracy better than $0.01^{\circ}$ are achieved in the real-time attitude tracking of the free-moving object. As an additional benefit, the real-time angular velocity and acceleration of all the attitude angles can be obtained simultaneously. These experimental results demonstrate that the proposed attitude metrology is ready to be applied to the real-time remote attitude sensing with high angular resolution and accuracy.
\end{abstract}

\section{INTRODUCITON}

Out of the six degrees of freedom for determining the motion of a free-moving object, the measurement of the three attitude angles (yaw $\varphi$, pitch $\theta$ and roll $\phi$ ) describing rotary motion are indispensable in the fields of navigation of the aircraft (Wang and Wang, 2019), robotics control (Liu et al., 2015) and precision manufacturing (Dong et al., 2016). At present, the popular attitude metrologies are commonly performed by combing the attitude sensor information (Crassidis and Markley, 2016; Zhi et al., 2018), visual image analysis (Cornall et al., 2006; Sim et al., 2002), laser autocollimation method (Saito et al., 2009; Chen et al., 2016), and so on. However, the systems utilizing the sensor information should be equipped with multiple sensors to accurately measure the attitude angles simultaneously, which will make the systems complicated and costly. The accuracy of the visual image analysis is greatly affected by the weather and environment. The laser autocollimation method has high sensitivity and accuracy, but its measurement range is limited. The above techniques cannot be applied to some special applications, such as the vibration monitoring of the machine tool (Goyal and Pabla, 2016) and the attitude calibration of the precision stages (Huang et al., 2018), which require the attitude metrology to have high sensitivity and accuracy in a large measurement range, as well as high temporal resolution and environmental adaptability. In comparison, ellipsometry is a powerful optical metrology technique that can obtain comprehensive optical information of both the layered and bulk materials (Liu et al., 2015). The attitude metrology based on the ellipsometry has the advantages of non-contact, flexibility, low cost, high accuracy and sensitivity, large measurement range, and shows great potential in the attitude metrology (Li et al., 2005).

Recently, more and more researchers have conducted detailed researches on the attitude metrology using ellipsometry. Chen et al. presented a roll-angle sensor based on the principle of polarization modulation by a quarter-wave plate (Chen et al., 2019). Although a notably large dynamic range of $0^{\circ} \sim 180^{\circ}$ and a resolution of $0.02^{\circ}$ are achieved, the angular accuracy is only $0.3^{\circ}$. Treichel et al. presented an attitude sensor using two perpendicular linearly polarized laser diodes for amplitude and polarization modulation, which has achieved an accuracy of 1 arcsec with the roll in the range of $\pm 30^{\circ}$, pitch and yaw in the range of $\pm 5^{\circ}$ (Treichel et al., 1999). However, the attitude angles are obtained separately under different instrument configurations. Zhang et al. combined the field-of-view effect of birefringent crystal with the high-speed polarimetry for real-time attitude tracking (Zhang et al., 2020), the method has an accuracy of $0.02^{\circ}$ with the roll in the range of $0^{\circ} \sim 360^{\circ}$, pitch and yaw in the theoretical range of $\pm 90^{\circ}$. However, the extraction of the attitude angle is performed under a specific polarization state of the incident light, which makes the measurement process cumbersome and introduces additional measurement errors. Meanwhile, since the attitude angles are extracted from the measured Stokes vector, it cannot eliminate the complex influence of unexpected polarization properties (such as depolarization, linear diattenuation caused by the light reflecting back and forth between the optical elements in the sensing unite) on the attitude metrology in the reflection mode. Then, the method is only suitable to be used in the transmission mode, which limits the application of the method. In contrast, the highspeed Mueller matrix ellipsometry (MME) is able to provide the complete Mueller matrix within several microseconds in each measurement, and thereby is capable of offering much more useful sample information such as anisotropy and depolarization in real time (Zhang et al., 2020). The attitude metrology based on the high-speed MME is no longer implemented under a specific polarization state of the incident light and can eliminate the errors caused by the unexpected polarization properties, which will improve the applicability, accuracy, sensitivity and robustness of the attitude measurement. These advantages make the high-speed

* Corresponding authors at: State Key Laboratory of Digital Manufacturing Equipment and Technology, Huazhong University of Science and Technology, Wuhan 430074, China (H. Jiang, H. Gu).

E-mail addresses: hjiang@hust.edu.cn (H. Jiang), hongganggu@ hust.edu.cn (H. Gu). 
MME a potential solution for the real-time remote attitude sensing.

In this work, we present an MME-based attitude sensor, which implements the attitude sensing from the measured attitudedependent Mueller matrix of a birefringent waveplate attached to the object. Since the proposed attitude metrology inherits the unique advantages of the high-speed MME, it is feasible for both transmission and reflection modes and can achieve real-time remote attitude tracking with high accuracy and robustness in an ultra-large measurement range, i.e., the roll in the range of $0^{\circ} \sim 360^{\circ}$, the theoretical ranges of the yaw and pitch are $-90^{\circ} \sim$ $90^{\circ}$, respectively. Through theoretical derivation of the principle, the mapping between the attitude angles and the Mueller matrix is constructed in the simulation experiments. In the dynamic experiments, the consistency between the measured and simulated Mueller matrices verifies the correctness of the proposed method. By using the high-speed MME with a temporal resolution of $11 \mu \mathrm{s}$, an angular resolution up to $0.00022^{\circ}$ and an angular accuracy better than $0.01^{\circ}$ have been achieved in the realtime remote attitude tracking of a rotating object. Additionally, the real-time angular velocity and acceleration of all the attitude angles can be accurately obtained at the same time. These realtime remote attitude tracking results demonstrate the advantages and applicability of the proposed metrology.

\section{EXPERIMENTAL SETUP}

The instrument used for the attitude metrology is a high-speed complete MME with a sub-microsecond temporal resolution. The MME consists of three parts: a polarization state generator (PSG), a sample stage and a polarization state analyzer (PSA), as shown in Fig. 1. The PSG contains a light source, a linear polarizer and two photoelastic modulators (PEMs), and can generate any polarization state. The light source is a $5 \mathrm{~mW}$ red $(632.8 \mathrm{~nm}) \mathrm{He}$ Ne laser (HNL050LB, THORLABS). The nominal frequencies of the two PEMs (II/FS47, II/FS50LR, Hinds) are $47.11 \mathrm{KHz}$ and $50.00 \mathrm{KHz}$ respectively, making the modulation frequency of PSG reach $100 \mathrm{KHz}$. The PSA is a six-parallel-channel full Stokes polarimeter, which can detect the full Stokes vector of a polarized light with an arbitrary polarization state in several nanoseconds (Zhang et al., 2018). The sample stage consists of a pitch angle adjustment stage, a yaw angle adjustment stage and a motorized rotation stage (PRM1Z8, THORLABS), which can continuously change the roll $\phi$ within the range of $0 \sim 360^{\circ}$, and fix the yaw $\varphi$ and pitch $\theta$ at an arbitrary value within the range among $\pm 30^{\circ}$ and $\pm 7^{\circ}$, respectively. The sensing unit in the transmission mode (SUT) is a quartz multi-order quarter-wave plate (WPMQ10M-633, THORLABS), while the sensing unit in the reflection mode (SUR) consists of a quartz multi-order quarter-wave plate with a reflective layer, a plano-convex lens and a 50:50 (R:T) nonpolarization beam splitter (NPBS). The MME is mounted on a rotatable base so that it can be easily switched between the transmission mode and the reflection mode.

\section{PRINCIPLE AND METHOD}

The quartz waveplate used in the attitude metrology is a uniaxial birefringent crystal, in which the two refractive indices $n_{\mathrm{e}}$ and $n_{\mathrm{o}}$ are in the directions parallel with and perpendicular to the optical axis, respectively. When a polarized light propagates through the uniaxial birefringent crystal, a phase difference $\delta$ will be introduced between the extraordinary and ordinary rays in the outgoing light (Wu et al., 1984), as shown in Fig. 2. And the $\delta$ can be calculated by Eq. (1)

$$
\delta=\frac{2 \pi}{\lambda} L
$$

where $\lambda$ is the vacuum wavelength of the incident light, $L$ is the optical path difference between the extraordinary and ordinary rays.
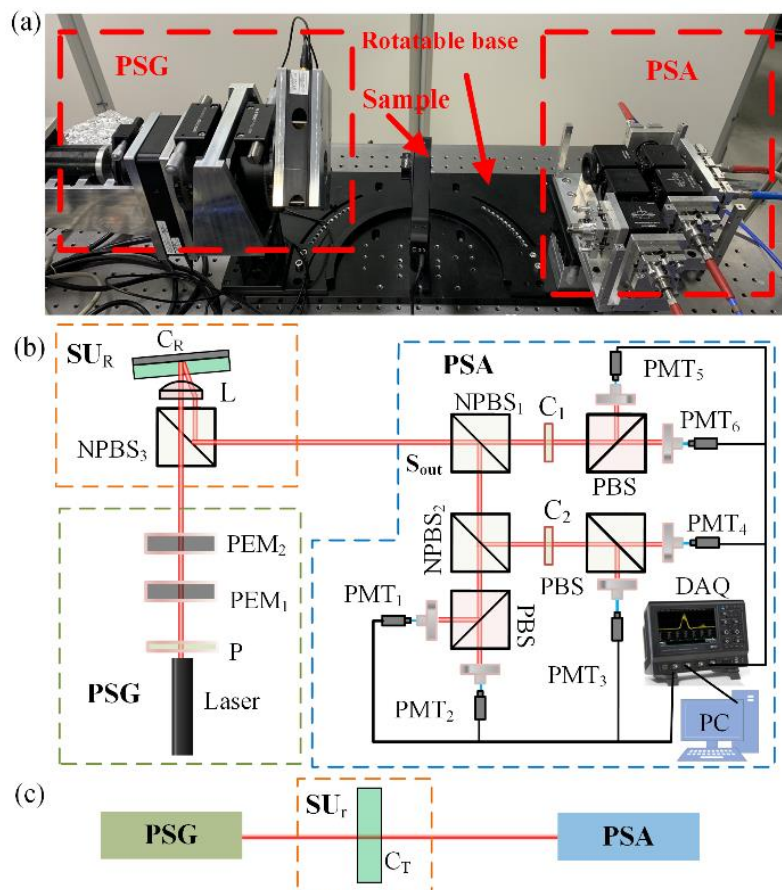

Figure 1. The high-speed $\bar{M}$ ME: $\mathrm{P}$ - polarizer; $\mathrm{PEM}_{1}$ and $\mathrm{PEM}_{2}$ - photoelastic modulators; $\mathrm{C}_{1}$ - quart-wave plate; $\mathrm{C}_{2}$ - half-wave plate; $C_{R}$ - quartz multi-order quart-wave plate with a reflective layer; $\mathrm{C}_{\mathrm{T}}$ - quartz multi-order quart-wave plate; $\mathrm{S}$-Sample; PBS - polarization beam splitter (PBS); NPBS 1 - 70:30 (R:T) non-polarization beam splitter (NPBS); $\mathrm{NPBS}_{2}, \mathrm{NPBS}_{3}-50: 50$ (R:T) NPBS; L - plano-convex lens; Ry- reflective layer; DAQ oscilloscope; PC - personal computer; PMT - photomultiplier tube; (a) the MME prototype, (b) light path diagram of the reflection mode; (c) light path diagram of the transmission mode;

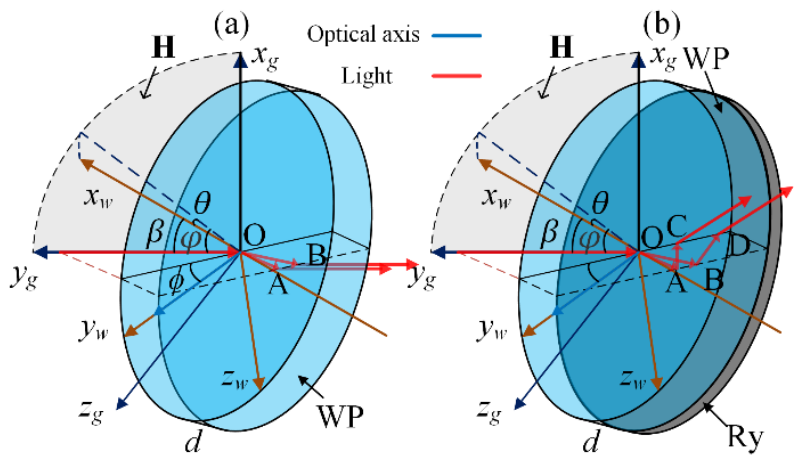

Figure 2. Schematic of the light propagation in the waveplate under arbitrary attitude angles, $x_{\mathrm{w}}-y_{\mathrm{w}}-z_{\mathrm{w}}$ is the coordinate system of the waveplate, $x_{\mathrm{g}}-y_{\mathrm{g}}-z_{\mathrm{g}}$ is the coordinate system of the ground.

$\mathbf{H}$ is the horizontal plane, which is composed of the $x_{\mathrm{g}}-y_{\mathrm{g}}$ axis. The $\phi$ refers to the azimuth of the incident plane with respect to the optical axis, which also represents the roll. The $\beta$ refers the incident angle of the input light. The angle between $x_{\mathrm{w}}$ axis and the projection of $x_{\mathrm{w}}$ on the $\mathbf{H}$ plane is the pitch $\theta$, and the angle

between the projection and $y_{\mathrm{g}}$ axis is the yaw $\varphi$. WP is the

birefringent waveplate; Ry is the reflective layer: (a) the transmission mode; (b) the reflection mode.

It is well known that the extraordinary index $n_{\mathrm{e}}$ will change 
with the incident angle in the uniaxial crystal, while the ordinary index $n_{\mathrm{o}}$ remains unchanged (Gu et al., 2017; Veiras et al., 2010). If the optical axis of the waveplate is parallel with the $y_{\mathrm{w}}$-axis, the effective refractive indices along $y_{\mathrm{w}}-z_{\mathrm{w}}$ axis can be expressed as

$$
\left\{\begin{array}{c}
n_{y}=n_{\mathrm{e}}\left[1+\left(\frac{1}{n_{\mathrm{e}}^{2}}-\frac{1}{n_{\mathrm{o}}^{2}}\right) \sin ^{2} \beta \cos ^{2} \phi\right]^{1 / 2} . \\
n_{z}=n_{\mathrm{o}}
\end{array}\right.
$$

According to the propagation of the light in the waveplate shown in Fig. 2, the optical path difference $L_{\mathrm{t}}$ and $L_{\mathrm{r}}$ in the transmission and reflection modes can be written as Eq. (3) and Eq. (4), respectively.

$$
\begin{gathered}
L_{t}=n_{y}|\mathrm{OA}|-n_{z}|\mathrm{OB}|-|\mathrm{AB}| \sin \beta \\
=d\left(\sqrt{n_{y}^{2}-\sin ^{2} \beta}-\sqrt{n_{z}^{2}-\sin ^{2} \beta}\right), \\
L_{r}=L_{1}(\phi, \beta)+L_{2}(-\phi, \beta) \\
=n_{y}|\mathrm{OA}|-n_{z}|\mathrm{OB}|+n_{y}|\mathrm{AC}|-n_{z}|\mathrm{BD}|-|\mathrm{CD}| \sin \beta \\
=2 d\left(\sqrt{n_{y}^{2}-\sin ^{2} \beta}-\sqrt{n_{z}^{2}-\sin ^{2} \beta}\right),
\end{gathered}
$$

where $d$ is the thickness of the waveplate. And it should be noted that the $\theta$ and $\varphi$ are coupled in the measurement of the incident angle $\beta$, where $\cos \beta=\cos \theta \cos \varphi$. In order to decouple them, the $\theta$ is measured by an electric level (HVT815T-30, MSENSOR TECHNOLOGY), which has a sampling rate of $100 \mathrm{~Hz}$ and an accuracy of $0.007^{\circ}$.

Combined with the above description and derivation, a theorical mapping between the attitude of the waveplate and its retardance can be established:

$$
\begin{aligned}
& \delta_{\mathrm{r}}(\beta, \phi)=2 \delta_{\mathrm{t}}(\beta, \phi)=\frac{2 \pi}{\lambda} L_{r} \\
& =\frac{4 \pi}{\lambda} d\left(\sqrt{n_{e}^{2}-\frac{n_{e}^{2} \cos ^{2} \phi+n_{o}^{2} \sin ^{2} \phi}{n_{o}^{2}} \sin ^{2} \beta}-\sqrt{n_{o}^{2}-\sin ^{2} \beta}\right) .
\end{aligned}
$$

When the polarized light is obliquely incident, the quartz waveplate exhibits not only linear birefringence (LB), but also weak linear diattenuation (LD) and circular birefringence (CB) (Arteaga et al., 2009; Gu et al., 2015). To construct the mapping between the attitude angles and the Mueller matrix, the polarization properties of the waveplate in the transmission and reflection modes are respectively expressed as:

$$
\begin{gathered}
\mathbf{M}_{\mathrm{WPT}}^{\mathrm{c}}(\phi, \beta, \psi, \gamma)=\mathbf{R}(-\phi) \mathbf{M}_{\mathrm{rd}}\left(\delta_{\mathrm{t}}, \psi\right) \mathbf{R}(\phi,) \mathbf{M}_{\mathrm{CB}}(\gamma), \\
\mathbf{M}_{\mathrm{WPR}}^{\mathrm{c}}(\phi, \beta, \psi, \gamma)=\mathbf{R}(\phi) \mathbf{M}_{\mathrm{rd}}\left(\delta_{r} / 2, \psi\right) \mathbf{R}(-\phi,) \mathbf{M}_{\mathrm{CB}}(\gamma) \ldots \\
\mathbf{M}_{\mathrm{Rf}} \mathbf{R}(-\phi) \mathbf{M}_{\mathrm{rd}}\left(\delta_{r} / 2, \psi\right) \mathbf{R}(\phi,) \mathbf{M}_{\mathrm{CB}}(\gamma),
\end{gathered}
$$

where the superscripts "c" represents the calculated matrix; LD is described with a diattenuation angle $\psi$ (Gu et al., 2018); the $\mathrm{CB}$ is described with an optical rotation angle $\gamma(\mathrm{Lo}, 2012)$. The muller matrix of the reflective layer $\mathbf{M}_{\mathrm{Rf}}$ is a diagonal matrix, whose diagonal elements are $1,1,-1$, and -1 . Meanwhile, the forms of the undefined matrix are:

$$
\mathbf{M}_{\mathrm{rd}}(\delta, \psi)=\left[\begin{array}{cccc}
1 & -\cos (2 \psi) & 0 & 0 \\
-\cos (2 \psi) & 1 & 0 & 0 \\
0 & 0 & \sin (2 \psi) \cos \delta & \sin (2 \psi) \sin \delta \\
0 & 0 & -\sin (2 \psi) \sin \delta & \sin (2 \psi) \cos \delta
\end{array}\right],(8)
$$$$
\mathbf{R}(x)=\mathbf{M}_{\mathrm{CB}}(x)=\left[\begin{array}{cccc}
1 & 0 & 0 & 0 \\
0 & \cos (2 x) & \sin (2 x) & 0 \\
0 & -\sin (2 x) & \cos (2 x) & 0 \\
0 & 0 & 0 & 1
\end{array}\right], x=\phi, \gamma .
$$

In principle, the light intensity matrix B measrued by six PMTs can be obtained by multiplying the modulation matrix $\mathbf{W}$ of PSG, the Mueller matrix of the sample $\mathbf{M}_{\mathrm{wpt}}$ and the demodulation matrix A of the PSA (De Martino et al., 2003) in the transmission mode:

$$
\mathbf{B}=\mathbf{A} \mathbf{M}_{\mathrm{WPT}}^{\mathrm{m}} \mathbf{W}
$$

where the superscripts " $m$ " represents the measured matrix; the demodulation matrix $\mathbf{A}$ is obtained by the in-situ calibration process of the PSA (Zhang et al., 2018). The modulation matrix W is composed of the Stokes vector of the incident polarized light.

In comparison, in order to ensure that the light reflected from the tilted waveplate can be detected by the PSA in the reflection mode, a NPBS and a plano-convex lens are introduced in the $\mathrm{SU}_{R}$ for light collimation. And the collimation is achieved by the incident light passing through the lens along the optic axis and then reflecting from the focal point of the lens, as shown in Fig.3. Since there are additional optical elements in the reflection mode, the matrix form in the flection mode is rewritten as:

$$
\mathbf{B}=\mathbf{A} \mathbf{M}_{\mathrm{BSR}} \mathbf{M}_{\mathrm{L}} \mathbf{M}_{\mathrm{WPR}}^{\mathrm{m}} \mathbf{M}_{\mathrm{BST}} \mathbf{W},
$$

where $\mathbf{M}_{\mathrm{BSR}}$ and $\mathbf{M}_{\mathrm{BST}}$ are the reflection and transmission Mueller matrix of the NPBS, which can be in-situ calibrated before the attitude tracking (Zhang et al., 2018). $\mathbf{M}_{\mathrm{L}}$ is the Mueller matrix of the plano-convex lens.

Since the polarization effect of the lens will introduce errors to the attitude metrology, a calibration method is proposed for the lens. In order to reduce the influence of the polarization effect of the lens on the attitude metrology, the lens used should be made of isotropic material. In this way, the polarization effect of the lens mainly comes from the surface, while the polarization effect of the internal space can be ignored.

According to the propagation trajectory of light reflected from the waveplate shown in Fig. 3, we can obtain the following angular relationship with geometric optics and Fresnel equation:

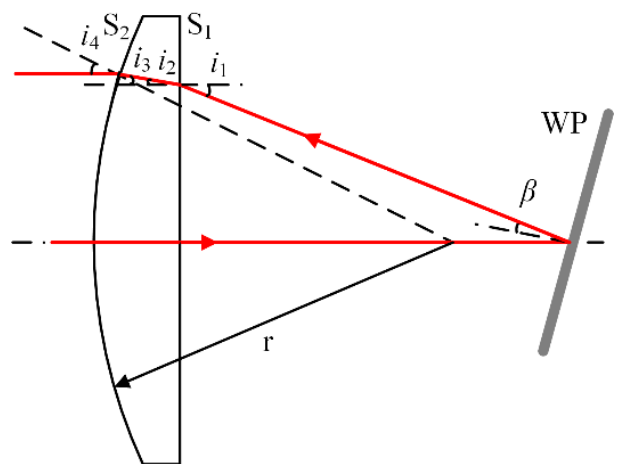

Figure 3. Schematic of the light propagation in the planoconvex lens: $r$ is lens surface radius, WP - waveplate with reflective layer; $S_{1}$ and $S_{2}$ - surface of the lens. 


$$
n_{\mathrm{L}}=\frac{\sin i_{1}}{\sin i_{2}}=\frac{\sin i_{4}}{\sin i_{3}} ; i_{4}=i_{2}+i_{3} ; i_{1}=2 \beta .
$$

where $n_{\mathrm{L}}$ is the refractive index of the internal space of the lens. When the reflected light passes through the surfaces $S_{1}$ and $S_{2}$ of the lens, the transmission coefficients of the $\mathrm{p}$ - and s-components of the light are $t_{\mathrm{p} 1}, t_{\mathrm{p} 2}, t_{\mathrm{s} 1}$ and $t_{\mathrm{s} 2}$ respectively, which are defined as:

$$
\begin{aligned}
& t_{\mathrm{p} 1}(\beta)=\frac{2 \cos i_{1}}{n_{\mathrm{L}} \cos i_{1}+\cos i_{2}} ; t_{\mathrm{s} 1}(\beta)=\frac{2 \cos i_{1}}{\cos i_{1}+n_{\mathrm{L}} \cos i_{2}} ; \\
& t_{\mathrm{p} 2}(\beta)=\frac{2 n \cos i_{3}}{\cos i_{3}+n_{\mathrm{L}} \cos i_{4}} ; t_{\mathrm{s} 2}(\beta)=\frac{2 n \cos i_{3}}{n_{\mathrm{L}} \cos i_{3}+\cos i_{4}} .
\end{aligned}
$$

Then, the Mueller matrix of the lens under arbitrary incident angle can be expressed as:

$$
\mathbf{M}_{\mathrm{L}}(\beta)=\left[\begin{array}{cccc}
1 & \sin \eta & 0 & 0 \\
\sin \eta & 1 & 0 & 0 \\
0 & 0 & \cos \eta & 0 \\
0 & 0 & 0 & \cos \eta
\end{array}\right]
$$

where $\eta$ is defined as:

$$
\tan \eta=\frac{t_{p 1}^{2} t_{p 2}^{2}-t_{s 1}^{2} t_{s 2}^{2}}{2 t_{p 1} t_{p 2} t_{s 1} t_{s 2}}
$$

It should be noted that the lens loses its polarization effect when the light passes along the optical axis of the lens, i.e. the $\mathbf{M}_{\mathrm{L}}$ becomes an identity matrix.

Based on the above derivation, the measured Mueller matrix of the waveplate under arbitrary attitude angles in the transmission mode and the reflection mode can be obtained from the detected light intensity:

$$
\begin{gathered}
\mathbf{M}_{\mathrm{WPT}}^{\mathrm{m}}=\mathbf{A}^{-1} \mathbf{B} \mathbf{W}^{-1}, \\
\mathbf{M}_{\mathrm{WPR}}^{\mathrm{m}}=\left(\mathbf{A M}_{\mathrm{BSR}} \mathbf{M}_{\mathrm{L}}\right)^{-1} \mathbf{B}\left(\mathbf{M}_{\mathrm{BST}} \mathbf{W}\right)^{-1},
\end{gathered}
$$

Finally, a least-squares regression analysis (LSRA) is introduced to obtain the measurand vector $\mathbf{x}$, which is defined as $\mathbf{x}=(\phi, \beta, \psi, \gamma)$ :

$$
\hat{\mathbf{x}}=\arg \min _{\mathbf{x} \subset \boldsymbol{\Omega}}\left\{\sum_{i=0}^{3} \sum_{j=0}^{3}\left[m_{i j}^{m}-m_{i j}^{c}(\mathbf{x})\right]^{2}\right\}^{1 / 2},
$$

where $m_{i j}$ is element in the $i$-th raw and $j$-th column of the Mueller matrix. $\boldsymbol{\Omega}$ denotes the associated parameter domain of the $\mathbf{x}$, where the parameter domains are $0^{\circ} \leq \phi \leq 360^{\circ},-90^{\circ}<\beta<90^{\circ}$, $-90^{\circ} \leq \psi \leq 90^{\circ}$ and $-180^{\circ} \leq \gamma \leq 180^{\circ}$ respectively.

Since the pitch $\theta$ is measured by an electric level in real-time, and the incident angle $\beta$ can be extracted from the measured Mueller matrix, then the yaw $\varphi$ can be calculated by

$$
\varphi(t)=\arccos \left[\frac{\cos \beta(t)}{\cos \theta(t)}\right]
$$

As an additional benefit, the real-time angular velocity $\omega$ and acceleration $\alpha$ of the attitude angles can be extracted simultaneously:

$$
\begin{gathered}
\omega(t)=\frac{x(t+\Delta t)-x(t)}{\Delta t}, x=\phi, \theta, \varphi . \\
\alpha(t)=\frac{\omega(t+\Delta t)-\omega(t)}{\Delta t} .
\end{gathered}
$$

\section{NUMERICAL SIMULATION}

A numerical simulation based on the above theoretical derivation is carried out to explore the change in the Mueller matrix of the birefringent waveplate versus attitude angles $(\phi, \beta)$. It should be noted that the proposed attitude metrology is theoretically feasible with the $\phi$ in range of $0^{\circ} \sim 360^{\circ}$, the $\theta$ and $\varphi$ in the range of $-90^{\circ} \sim 90^{\circ}$. However, limited by the clear aperture (CA) of the waveplate and lens and the travel of the adjustment stages, our method can only be demonstrated with $\theta$ in the range of $-7^{\circ}$ and $7^{\circ}$, and $\varphi$ in the range of $-30^{\circ} \sim 30^{\circ}$ for the practical experimental configurations. Therefore, the simulation experiment is performed in the range $0^{\circ} \sim 360^{\circ}$ for $\phi$ and $-30^{\circ} \sim 30^{\circ}$ for $\beta$. Since $\mathrm{LD}$ and $\mathrm{CB}$ are usually weak when $\beta$ is small, the $\psi=45^{\circ}$ and $\gamma=0^{\circ}$ are set in the simulation. Simulation results of the transmission and the reflection modes are shown in Fig. 4.
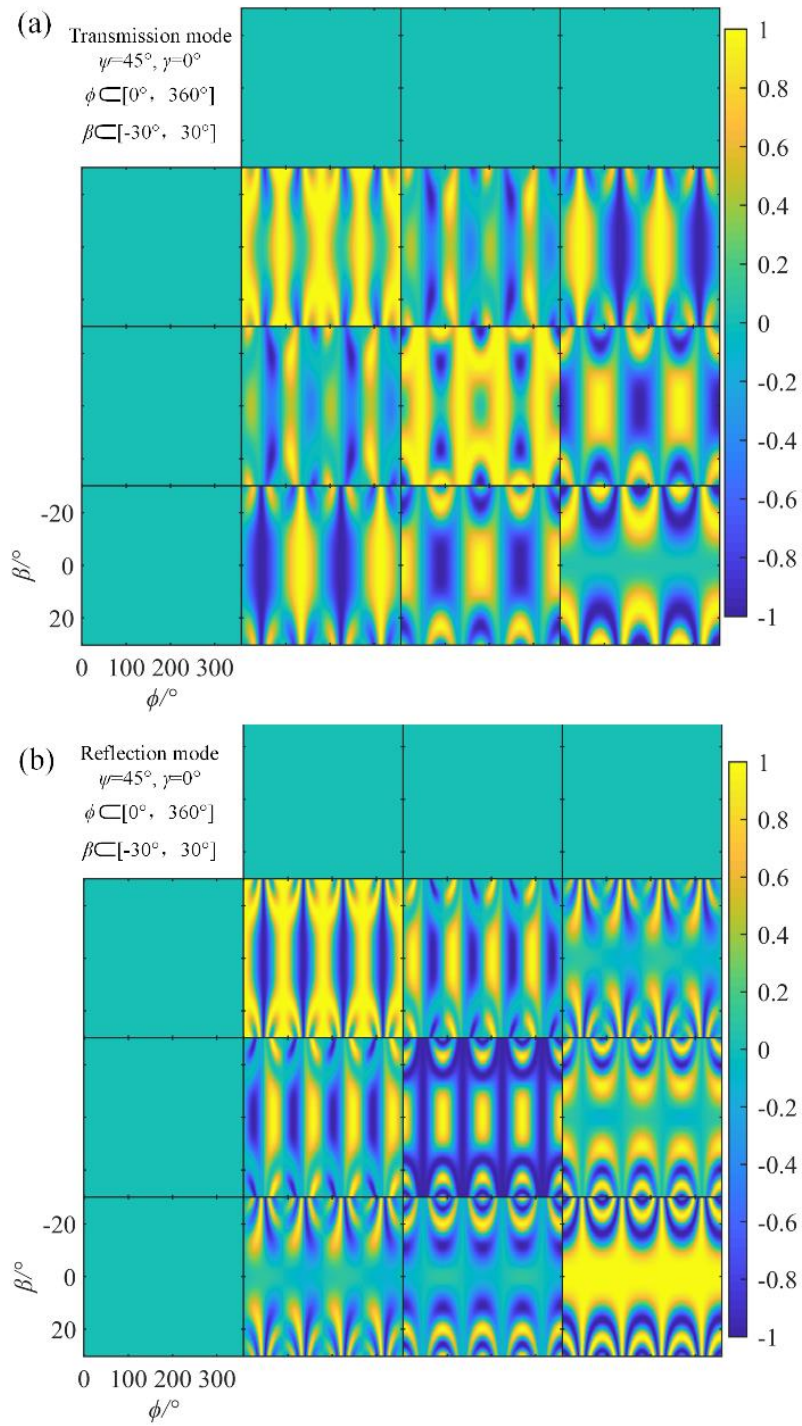
Figure 4 Normalized Muller matrix of the birefringent waveplate under different attitude angles $(\phi, \beta)$ : (a) transmission mode; (b) reflection mode.

Figure 4 shows the normalized Mueller matrix of the birefringent waveplate under different roll $\phi$ and incident angle $\beta$. In both of the transmission and reflection modes, the Mueller matrix elements in the first row and the first column are zero. However, it does not mean that they do not vary with the attitude angle. In the practical birefringent device, as the $\beta$ deviates from $0^{\circ}$, the LD and $\mathrm{CB}$ will be stronger and stronger, i.e. $\psi$ deviates from $45^{\circ}$ with an amplitude of about $0.5^{\circ}, \gamma$ deviates from $0^{\circ}$ with an amplitude of about $1^{\circ}$. These deviations will cause the elements vary between -0.02 and 0.02 , which should be handled carefully in the accurate attitude metrology.

In contrast, the elements of the $3 \times 3$ matrix block in the lower right corner regularly deviate from -1 to 1 versus the attitude angle. The variation of the Mueller matrix is symmetric about $\beta=0^{\circ}$. And when the $\beta$ is in the range of $-5^{\circ} \sim 5^{\circ}$, the Mueller matrix remains almost unchanged with the $\beta$, indicating a low sensitivity of the attitude angle measurement in this interval. The sensitivity in this interval can be improved by optimizing the thickness of the waveplate. When the $\beta$ is outside the range of $-5^{\circ} \sim 5^{\circ}$, the variations of the $3 \times 3$ matrix block elements are more and more intense as the absolute value of $\beta$ increases. Besides, when $\beta$ is constant, the Mueller matrix elements change periodically with the roll $\phi$. The variation period is $180^{\circ}$ in the transmission mode and $90^{\circ}$ in the reflection mode, respectively. The difference in the variation periods is caused by the different optical path differences in these two modes.

\section{EXPERIMENT AND DISCUSSION}

In this section, the correctness of the proposed metrology is firstly proved by the consistency between the theoretical prediction results and the actual measurement results in the normal incidence dynamic experiment in the transmission mode. Moreover, the successful application of the proposed metrology in the variable velocity oblique incidence experiment has demonstrated its applicability. The results achieved in the dynamic experiments of the transmission mode demonstrate the feasibility and robustness of the proposed method in the real-time attitude measurement of the free-moving object. Although only dynamic experiments in the transmission mode were implemented (dynamic experiments in the reflection mode are postponed due to the COVID-19), it can be expected that the proposed metrology can achieve considerable results according to the presented experimental setup and theoretical derivation.

\subsection{Normal incidence dynamic experiment}

The most specific example, namely the attitude measurement under the normal incidence in the transmission mode, is chosen to verify the correctness of the proposed metrology. In the dynamic experiment, the yaw $\varphi$ and pitch $\theta$ are fixed at $0^{\circ}$, and the roll $\phi$ driven by the motorized rotation stage continuously changes with an angular velocity of $20 \%$ s. The sampling rate of the attitude measurement is about $91.33 \mathrm{KHz}$. The Muller matrix of the waveplate versus time (blue solid line) is shown in Fig. 5.

It can be observed from Fig. 5 that the Mueller matrix elements in the first row and the first column nearly remain unchanged with $\phi$, while the $3 \times 3$ matrix block in the lower right corner regularly change with $\phi$. Combined with the theory derivation in section 3 , the LSRA shown in Eq. (18) is used to extract the measurands vector $\mathbf{x}$ from the Muller matrix over time as shown in Fig. 6 . Then, we can calculate the simulated Muller matrix with extracted $\mathbf{x}$, which is shown in the red dotted line in Fig. 5. It can be seen that the measured Mueller matrix is highly consistent with the calculated one, which indicates the correctness of proposed method.

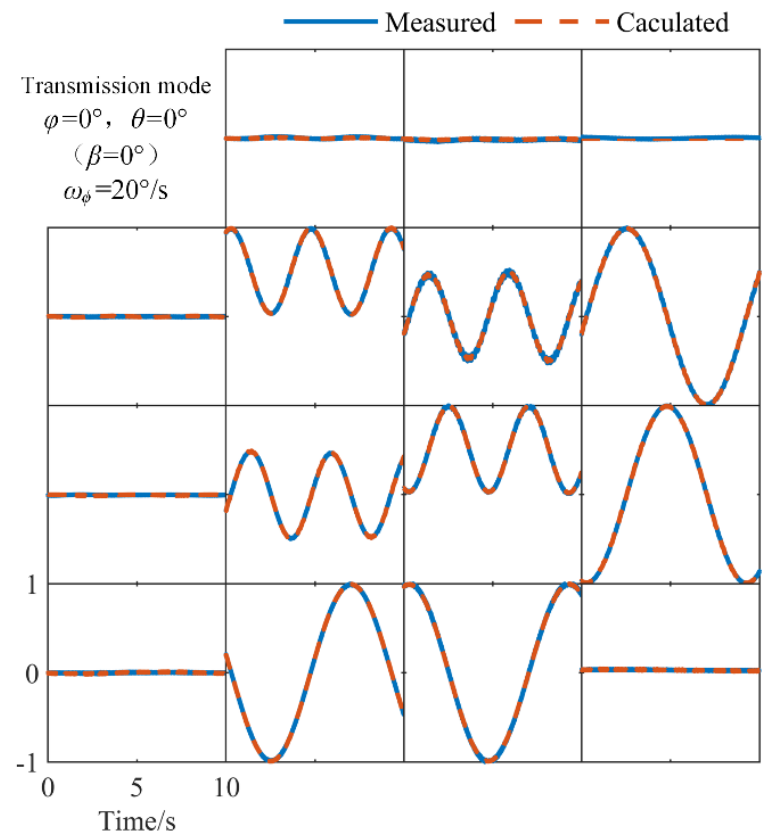

Figure 5 Normalized Muller matrix of the waveplate versus time in the normal incidence dynamic experiment in the transmission mode.
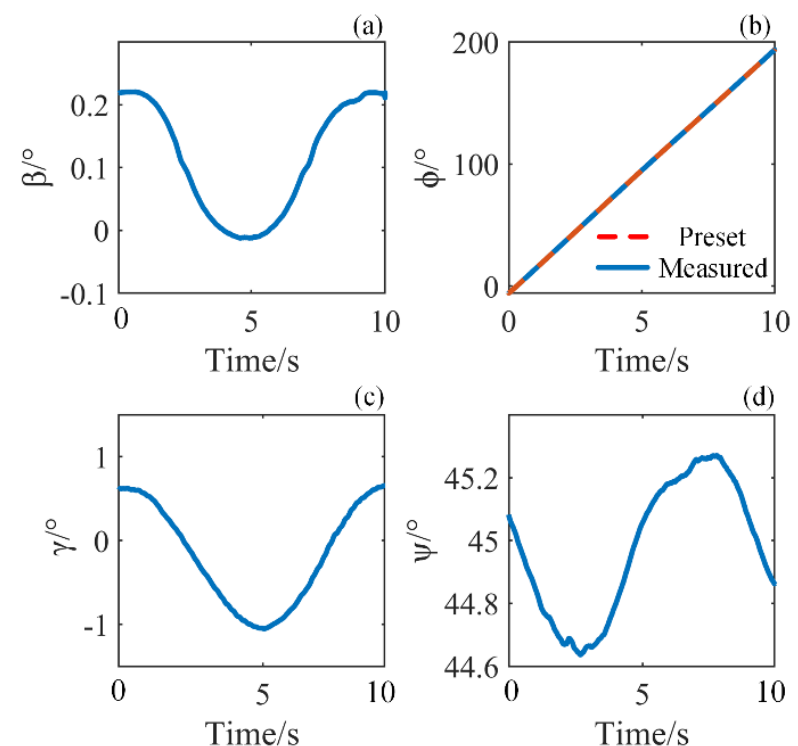

Figure 6 The measurands extracted from the Muller matrix in the normal incidence experiments: (a) incident angle $\beta$ over

time; (b) the preset and measured roll $\phi$ over time; (c) the optical rotation angle $\gamma$ over time; (d) the polarimetric angle $\psi$ over time.

As shown in Fig. 6(a), the incident angle $\beta$ does not remain at $0^{\circ}$ as expected, but fluctuates between $-0.01^{\circ}$ and $0.22^{\circ}$. The small regular fluctuation may be caused by the nonideal normal incidence with human operation and the misalignment of the waveplate rotation axis and the motorized stage rotation axis. And it can be observed from Fig. 6(b) that the roll $\phi$ increases linearly from $-5^{\circ}$ to $195^{\circ}$, which is highly consistent with the preset process. Meanwhile, as expected from the simulation 
experiments, the optical rotation angle $\gamma$ fluctuates around $0^{\circ}$ with an amplitude not exceeding $1^{\circ}$, and the diattenuation angle $\psi$ deviates from $45^{\circ}$ with an amplitude not exceeding $0.5^{\circ}$, which proves that the $\mathrm{LD}$ and $\mathrm{CB}$ properties are weak when $\beta$ is small.

Additionally, combined with the measured results of $\beta$ and $\phi$, we obtain the retardance $\delta$ of the waveplate over time with Eq. (5), and the results are shown in Fig. 7(a). The retardance is about $88^{\circ}$ instead of $90^{\circ}$, which may be due to the thickness error of the waveplate and the surface defects. Since the retardance $\delta$ of the waveplate will not change with the $\phi$ under normal incidence theoretically, the small variation in $\delta$ is caused by the small deviation in the $\beta$ shown in Fig. 6(a). Besides, an angular resolution analysis is performed to demonstrate the angular accuracy and sensitivity of the presented attitude metrology. It can be observed that the error between the preset and measured $\phi$ shown in Fig. 7(b) fluctuates between $\pm 0.01^{\circ}$. Since the MMEbased attitude metrology is a point-to-point angle measurement, the error should not be cumulative, and it is most likely caused by the unstable rotation of the motor. Meanwhile, the measured angular resolution of the $\phi$ varies around the preset value $0.00022^{\circ}$ with an amplitude deviation not exceeding $0.00001^{\circ}$, which indicates a high angular sensitivity of the proposed method. It should be noted that the angular accuracy and sensitivity is not only limited by the proposed attitude metrology, but also related to the performance of the motorized stage used in the experiments. Therefore, further improvements in the motor and motor control system may help us achieve better angular sensitivity and accuracy.

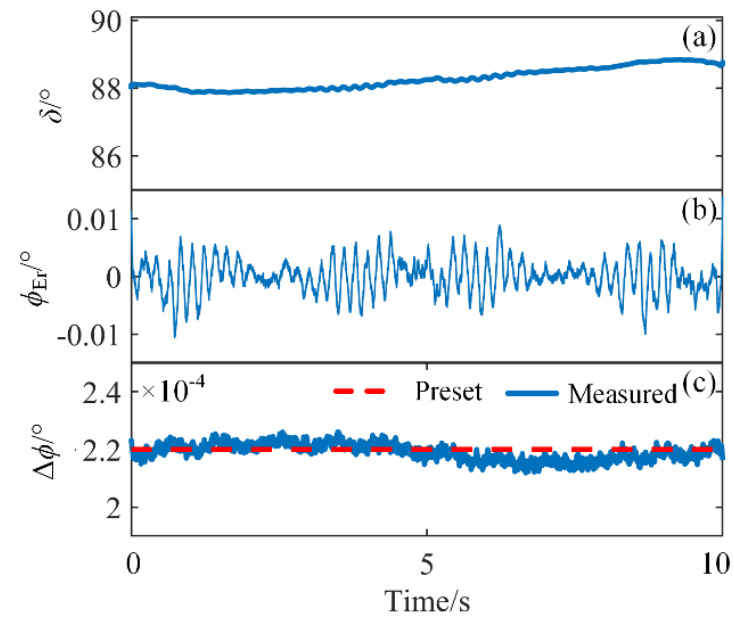

Figure 7 Relative results in the normal incidence experiment: (a) retardance $\delta$ over time; (b) measurement error $\phi_{\mathrm{Er}}$ over time, in which $\phi$ Er is defined as the preset results minus the measured results. (c) the measured and preset resolution $\Delta \phi$ over time, in which $\Delta \phi(t)=\phi(t+\Delta t)-\phi(t), \Delta t=11 \mu \mathrm{s}$

\subsection{Oblique incidence dynamic experiment}

On the premise of maintaining versatility, we performed an oblique incidence dynamic experiment under arbitrary attitude angles within the measurement range limited by the hardware. As an example, the pitch $\theta$ and yaw $\varphi$ are firstly fixed at $6^{\circ}$ and $20^{\circ}$ by the adjustment stages respectively, and the angular velocity $\omega_{\phi}$ of roll varies between $0 \% \mathrm{~s}$ and $20 \% \mathrm{~s}$ with a maximum acceleration of $20 \% \mathrm{~s}^{2}$. Meanwhile, to simulate the actual attitude of a free-moving object as realistically as possible, we simultaneously rotate the yaw angle adjustment stage manually, which makes the incident angle $\beta$ vary with the $\phi$. The sampling rate of the attitude measurement is still about $91.33 \mathrm{KHz}$. The Muller matrix of the waveplate versus time is shown with the blue solid line in the Fig. 8. And the extracted measurand vector $\mathbf{x}$ of the oblique incidence experiment is shown in Fig. 9.

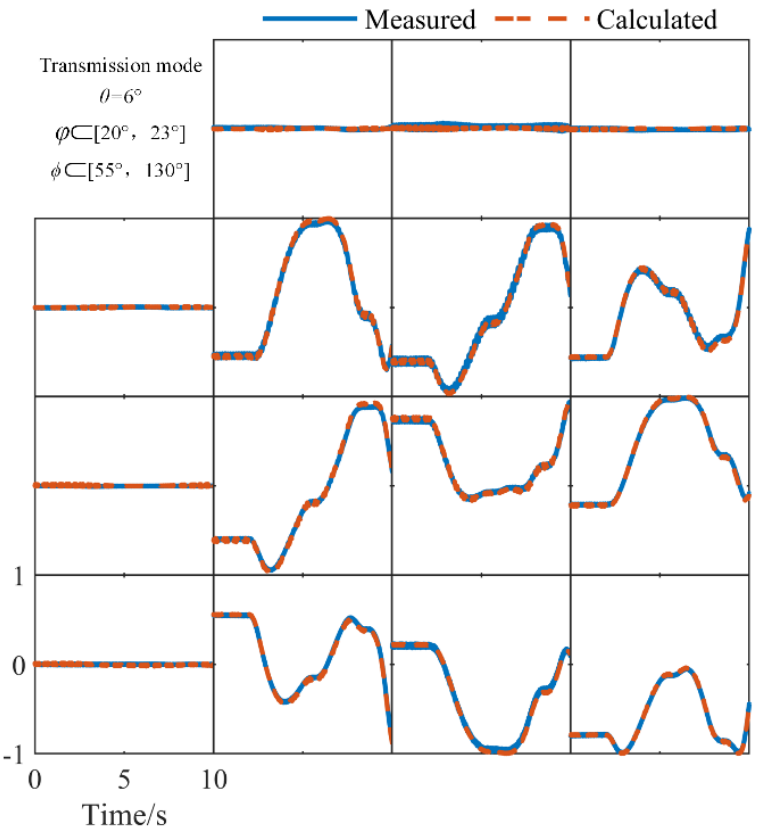

Figure 8 Normalized Muller matrix of the waveplate over time in the oblique incidence dynamic experiment.

It can be observed from Fig. 8 that the Mueller matrix elements in the first row and the first column still fluctuate around 0 , while the variation of the $3 \times 3$ matrix block in the lower right corner with $\phi$ is no longer regular. Then, the simulated Muller matrix reconstructed with the measurand vector $\mathbf{x}$ is shown with the red dotted line in the Fig. 8. The consistency between the measured and calculated matrix indicates that the proposed attitude metrology is still effective in the complex motion process.
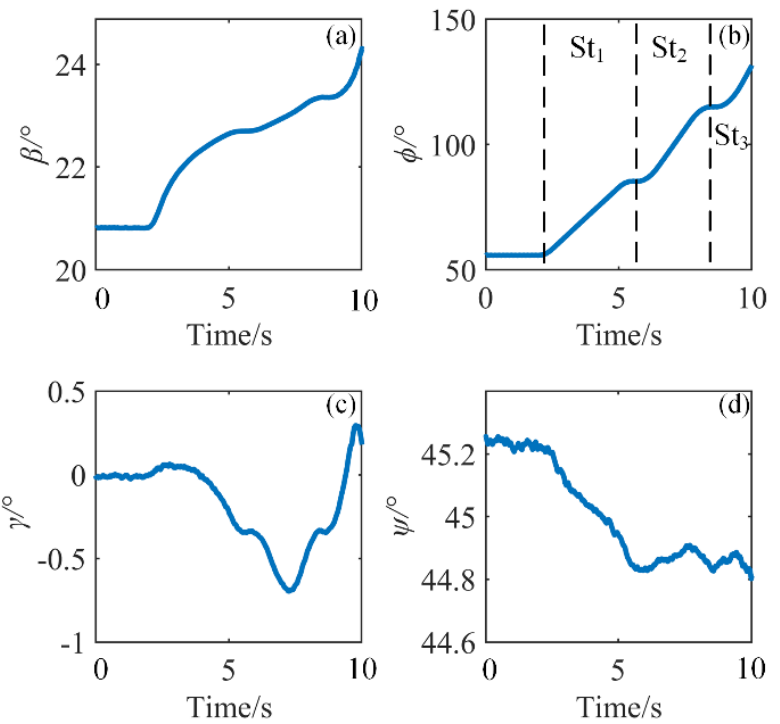

Figure 9 The measurands extracted from the Muller matrix in the oblique incidence experiments: (a) incident angle $\beta$ over time; (b) the measured roll $\phi$ over time; (c) the optical rotation

angle $\gamma$ over time; (d) the polarimetric angle $\psi$ over time.

As shown in Fig. 9(a), the $\beta$ increases irregularly from $20.81^{\circ}$ to $24.22^{\circ}$ when the $\theta$ is fixed and the $\varphi$ is changed manually. And the small deviations in the $\gamma$ and $\psi$ shown in the Fig. $9(\mathrm{c})$ and $9(\mathrm{~d})$ indicate the existence of weak CB and LD properties. Meanwhile, to achieve a detailed analysis of the change process of $\phi$ shown 
in Fig. 9(b), we calculate the angular velocity $\omega_{\phi}$ and acceleration $\alpha_{\phi}$ of roll with Eq. (20) and Eq. (21), and the results are shown in Fig. 10(a) and 10(b). Combined with results of $\phi, \omega_{\phi}$ and $\alpha_{\phi}$, the regular variation of the $\phi$ can be roughly divided into three stages $\mathrm{St}_{i}(i=1,2,3)$. In the first two stages $\mathrm{St}_{1}$ and $\mathrm{St}_{2}$, when the $\phi$ increases by $30^{\circ}$, the $\omega_{\phi}$ undergoes a trapezoidal change, while the $\omega_{\phi}$ increases from $0 \% \mathrm{~s}$ to the maximum velocity and then maintain the maximum velocity in the last stage $\mathrm{St}_{3}$. The maximum $\omega_{\phi}$ are $10 \% \mathrm{~s}, 15 \%$ and $20 \% \mathrm{~s}$ in the three stages respectively. And the $\alpha_{\phi}$ in the acceleration or deceleration process also undergoes a trapezoidal change, the maximum $\alpha_{\phi}$ is $20^{\circ} / \mathrm{s}^{2}$ in all three stages. Here, the acceleration time required for the parameter value from 0 to the maximum is defined as $t_{\mathrm{ac}}$, while the deceleration time required for the parameter value from the maximum to 0 is defined as $t_{\mathrm{dc}}$. It can be observed that the $t_{\mathrm{ac}}$ and $t_{\mathrm{dc}}$ in the change of the $\omega_{\phi}$ and $\alpha_{\phi}$ are equal. Besides, the $t_{\mathrm{ac}}$ of $\omega_{\phi}$ increases with the increase of the maximum $\omega_{\phi}$, while the $t_{\mathrm{ac}}$ of $\alpha_{\phi}$ remains unchanged at about $0.23 \mathrm{~s}$. The preset change of $\phi$ is accurately restored in detail with the proposed method.

Since the $\theta$ and $\varphi$ are coupled in the $\beta$, the $\theta$ is measured by an electric level. Because the sampling rate of the electric level is much lower than that of the high-speed MME, the piecewise linear interpolation is used to achieve the nominal sampling rate matching. As shown in Fig. 10 (c), the $\theta$ increases from $6^{\circ}$ to $6.07^{\circ}$ which may be due to the small misalignment of the waveplate rotation axis and the motorized stage rotation axis. Since the increment is rather small, it is meaningless to present the angular velocity and acceleration of the $\theta$ here. Combined with the measured $\beta$ and $\theta$, we can obtain the $\varphi$ over time with Eq. (19), and the results are shown in Fig. 10(d). It can be observed that the manually driven $\varphi$ experiences three different increments $\mathrm{Ic}_{i}$ $(i=1,2,3)$ as shown in Fig. 10(d f). In the first increment $\mathrm{Ic}_{1}$, the $\varphi$ increases from $20^{\circ}$ to $21.9^{\circ}$, and the angular velocity of yaw $\omega_{\varphi}$ first quickly increases to $1.45 \%$ and then slowly decreases to $0 \% \mathrm{~s}$ while the acceleration $\alpha_{\varphi}$ first quickly increases to $4.15 \% \mathrm{~s}^{2}$ and then quickly decreases to $-1.8 \% \mathrm{~s}^{2}$. In the second increment $\mathrm{Ic}_{2}$, the $\varphi$ increases from $21.9^{\circ}$ to $22.6^{\circ}$, and the angular velocity of yaw $\omega_{\varphi}$ first slowly increases to $0.39^{\circ} / \mathrm{s}$ and then slowly decreases to $0 \% \mathrm{~s}$, while the acceleration $\alpha_{\varphi}$ fluctuates around $0.38^{\circ} / \mathrm{s}^{2}$. In the last increment $\mathrm{Ic}_{3}$, the $\varphi$ increases from $22.6^{\circ}$ to $23.55^{\circ}$, the angular velocity of yaw $\omega_{\varphi}$ increases from $0 \%$ s to $1.82 \%$, and the acceleration $\alpha_{\varphi}$ increases from $0 \% \mathrm{~s}^{2}$ to $3.6 \% \mathrm{~s}^{2}$. The complex change of the manually driven $\varphi$ has been captured with the proposed method.
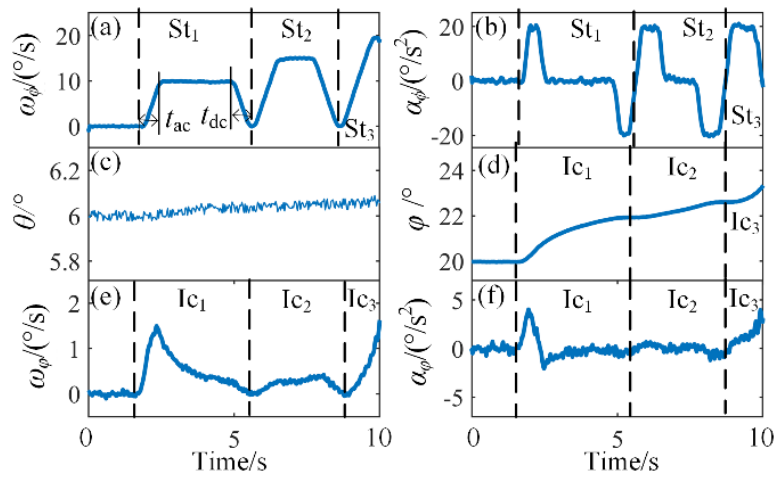

Figure 10 Relative results of attitude angle in the oblique incidence experiment: (a) angular velocity of roll $\omega_{\phi}$ over time; (b) angular acceleration of roll $\alpha_{\phi}$ over time; (c) the pitch $\theta$ over time; (d) the yaw $\varphi$ over time; (e) angular velocity of yaw $\omega_{\varphi}$ over time; (f) angular acceleration of yaw $\alpha_{\varphi}$ over time.

In the oblique incidence dynamic experiment, not only all the attitude angles can be captured accurately in real-time with the proposed attitude metrology, but also the real-time angular velocity and acceleration of all the attitude angles can be obtained simultaneously. The results and discussion demonstrate the applicability and robustness of the proposed method in the realtime remote attitude monitoring of the free-moving object.

\section{CONCLUTION}

A novel optical sensor is presented for real-time remote attitude tracking of the free-moving object. The sensor utilizing the highspeed MME achieves attitude sensing from the attitudedependent Mueller matrix of a birefringent waveplate attached to the object. With the unique advantages brought by the high-speed MME, the proposed attitude metrology is no longer implemented under a specific polarization state of the incident light, which can eliminate the errors introduced by the unexpected polarization properties. These advantages make the method applicable to an ultra-large measurement range with high accuracy and robustness in both of the transmission and the reflection modes. The basic principle is introduced via the theoretical derivation and the mapping between the Muller matrix of the waveplate and the attitude angles is constructed in the simulation experiments. Through the dynamic experiments in the transmission mode, the correctness of the proposed method is firstly verified by the consistency between the measured and calculated Mueller matrix Then, benefitting from the high temporal resolution and accuracy of the high-speed MME, an angular resolution up to $0.00022^{\circ}$ and an angular accuracy better than $0.01^{\circ}$ are achieved in the realtime remote attitude tracking of a rotating object. Additionally, the real-time angular velocity and acceleration of all the attitude angles can be obtained simultaneously. It should be noted that although only dynamic experiments in transmission mode are currently performed, according to the experimental setup and theoretical derivation presented, considerable results can also be achieved for the dynamic experiments in the reflection mode, which will be carried out in the near further after the COVID-19. In conclusion, results achieved in the transmission mode are sufficient to prove the applicability and advantages of the proposed method in the real-time remote attitude sensing, such as the in-situ attitude calibration of the high-precision machines or the attitude monitoring of the high-speed moving object.

\section{ACKNOWLEDGEMENTS}

This research was supported by the National Natural Science Foundation of China (51975232, 51525502, 51727809, 51805193); National Key Research and Development Program of China (2017YFF0204705); Natural Science Foundation of Hubei Province (2018CFA057); National Major Science and Technology Projects of China (2017ZX02101006-004).

\section{REFERENCES}

Arteaga, O., Canillas, A., Jellison Jr, G.E., 2009: Determination of the components of the gyration tensor of quartz by oblique incidence transmission two-modulator generalized ellipsometry. Appl. Opt. 48, 5307-5317.

Chen, X., Liao, J., Gu, H., Shi, Y., Jiang, H., Liu, S., 2019: Proof of principle of an optical Stokes absolute roll-angle sensor with ultra-large measuring range. Sens. Actuators, A-Phys 291, 144149.

Chen, Y.-L., Shimizu, Y., Kudo, Y., Ito, S., Gao, W., 2016: Modelocked laser autocollimator with an expanded measurement range. Opt. Express 24, 15554-15569. 
Cornall, T.D., Egan, G.K., Price, A., 2006: Aircraft attitude estimation from horizon video. Electron. Lett. 42, 744-745.

Crassidis, J.L., Markley, F.L., 2016: Three-axis attitude estimation using rate-integrating gyroscopes. J. Guid. Control. Dyn. 39, 1513-1526.

De Martino, A., Kim, Y.-K., Garcia-Caurel, E., Laude, B., Drévillon, B., 2003: Optimized Mueller polarimeter with liquid crystals. Opt. Lett. 28, 616-618.

Dong, Y., Zhang, Y., Ai, J., 2016: Full-altitude attitude angles envelope and model predictive control-based attitude angles protection for civil aircraft. Aerosp. Sci. Technol 55, 292-306.

Goyal, D., Pabla, B., 2016: The vibration monitoring methods and signal processing techniques for structural health monitoring: a review. Arch Comput Method E 23, 585-594.

Gu, H., Chen, X., Shi, Y., Jiang, H., Zhang, C., Gong, P., Liu, S., 2018: Comprehensive characterization of a general composite waveplate by spectroscopic Mueller matrix polarimetry. Opt. Express 26, 25408-25425.

Gu, H., Chen, X., Zhang, C., Jiang, H., Liu, S., 2017: Study of the retardance of a birefringent waveplate at tilt incidence by Mueller matrix ellipsometer. J. Opt. 20, 015401.

Gu, H., Liu, S., Chen, X., Zhang, C., 2015: Calibration of misalignment errors in composite waveplates using Mueller matrix ellipsometry. Appl. Opt. 54, 684-693.

Huang, Y., Fan, K.-C., Sun, W., Liu, S., 2018: Low cost, compact 4-DOF measurement system with active compensation of beam angular drift error. Opt. Express 26, 17185-17198.

Li, S., Yang, C., Zhang, E., Jin, G., 2005: Compact optical rollangle sensor with large measurement range and high sensitivity. Opt. Lett. 30, 242-244.

Liu, H., Wang, X., Zhong, Y., 2015: Quaternion-based robust attitude control for uncertain robotic quadrotors. IEEE $T$ Ind Inform 11, 406-415.

Liu, S., Chen, X., Zhang, C., 2015: Development of a broadband Mueller matrix ellipsometer as a powerful tool for nanostructure metrology. Thin Solid Films 584, 176-185.

Lo, Y.-L., 2012: Extraction of effective parameters of turbid media utilizing the Mueller matrix approach: study of glucose sensing. J. Biomed. Opt. 17, 097002.

Saito, Y., Arai, Y., Gao, W., 2009: Detection of three-axis angles by an optical sensor. Sens. Actuators, A-Phys 150, 175-183.

Sim, D.-G., Park, R.-H., Kim, R.-C., Lee, S.U., Kim, I.-C., 2002: Integrated position estimation using aerial image sequences. IEEE T Pattern Anal 24, 1-18.

Treichel, R., Sesselmann, R., Krieger, J., 1999: Optical sensor for measurement of roll+ pitch+ yaw angles over large distances with high accuracy, Proc. SPIE 3824, Optical Measurement Systems for Industrial Inspection. International Society for Optics and Photonics, 199-207. doi.org/10.1117/12.364285.

Veiras, F.E., Perez, L.I., Garea, M.T., 2010: Phase shift formulas in uniaxial media: an application to waveplates. Appl. Opt. 49,

\section{$2769-2777$}

Wang, X., Wang, W., 2019: Extended signal-correction observer and application to aircraft navigation. IEEE $T$ Ind Electron 67,3149-3156.

Wu, S.-T., Efron, U., Hess, L.D., 1984: Birefringence measurements of liquid crystals. Appl. Opt. 23, 3911-3915.

Zhang, S., Gu, H., Liu, J., Jiang, H., Chen, X., Zhang, C., Liu, S., 2018: Characterization of beam splitters in the calibration of a six-channel Stokes polarimeter. J. Opt.20,125606.

Zhang, S., Jiang, H., Gu, H., Chen, X., Liu, S., 2020: Attitude metrology based on the field-of-view effect of birefringence using high-speed polarimetry. Opt. Lett. 45, 2074-2077.

Zhang, S., Jiang, H., Gu, H., Chen, X., Liu, S., 2020: High-speed Mueller matrix ellipsometer with microsecond temporal resolution. Opt. Express 28, 10873-10887.

Zhi, W., Chu, J., Li, J., Wang, Y., 2018: A novel attitude determination system aided by polarization sensor. Sensors 18 , 158. 